J. SIAM APPL, MATH.

Vol. 14, No. 5, September, 1966

Printed in U.S.A.

\title{
AN INTEGRAL TRANSFORM ASSOCIATED WITH BOUNDARY CONDITIONS CONTAINING AN EIGENVALUE PARAMETER*
}

\author{
DONALD S. COHEN†
}

1. Introduction. It has long been known that certain integral transforms and Fourier-type series can be used to solve many classical boundary and initial value problems in separable coordinate systems. More recently, it has been shown that these classical transforms and series are spectral representations associated with an ordinary differential system which results on applying separation of variables to the given boundary value problem. This has been the basis for recent work concerned with systematically generating the proper spectral representation needed to solve a given problem. See [1]-[4] for a list of references.

We shall consider the problem of finding the associated spectral representation when the resulting ordinary differential system has the eigenvalue parameter occurring in both the equation and one boundary condition. Moreover, the differential equation is to be satisfied on a semi-infinite interval, thus leading to a singular problem which does not seem to have been studied before. In $\$ 2$ by using a transformation due to B. Friedman (which we modify appropriately for our singular case), we first give a formal derivation of the spectral representation, and then we rigorously prove the result.

In $\S 3$ our representation is applied to solve an initial-boundary value problem arising in the theory of diffusion and heat flow in one dimension. We should bear in mind that even in cases where solutions are already known, our method systematically yields alternative representations which are often more rapidly convergent and from which asymptotic expansions of solutions with respect to parameters can often be found. In the problem to be considered a representation of the solution can also be found easily by a straight-forward application of the Laplace transform in time $t$, while the new transform derived in $\$ 2$ yields the solution when applied in space $x$. The new transform, however, can be applied when the coefficients in the boundary value problem are time dependent, a situation which, in

* Received by the editors February 10, 1966.

$\dagger$ Firestone Flight Sciences Laboratory, Graduate Aeronautical Laboratories, California Institute of Technology, Pasadena, California. This research was partially supported by the Air Office of Scientific Research under Grant AF-AFOSR-182-64 while the author was at Rensselaer Polytechnic Institute and partially supported by the National Science Foundation under Grant GP-4597 at the California Institute of Technology. 
general, precludes the use of the Laplace transform. As a general rule [1] there should be a spectral representation associated with each ordinary differential system resulting from applying separation of variables to the original boundary value problem, and each spectral representation should lead to a different representation of the solution of the original problem.

2. The spectral representation. We shall need the spectral representation associated with the following second order ordinary differential system:

$$
\begin{aligned}
u^{\prime \prime}(x)+\lambda u(x) & =0, \quad x \geqq 0, \\
u^{\prime}(0)-\lambda u(0) & =0, \\
\lim _{x \rightarrow \infty}|u(x)| & <\infty .
\end{aligned}
$$

We shall first obtain the desired representation by purely formal means; a rigorous verification is given immediately after this. We shall use a modification of a transformation due to B. Friedman [5], the modification being necessary since our problem is singular and that of Friedman was not.

Let $\delta$ be the space of two component vectors $U$ whose first component is a real twice differentiable function $u(x)$ on the open interval $x>0$ and whose second component is a scalar $u_{0}$; that is,

$$
U(x)=\left(\begin{array}{c}
u(x) \\
u_{0}
\end{array}\right) .
$$

Now, consider the subspace $\mathscr{D}$ of vectors $U \in \mathbb{S}$ such that $u_{0}=u(0)$ and such that $u(x)$ is bounded as $x \rightarrow \infty$. If we define an operator $L$, operating on vectors $U \in D$, as

$$
L U \equiv\left(\begin{array}{c}
-u^{\prime \prime}(x) \\
u^{\prime}(0)
\end{array}\right) .
$$

Then our eigenvalue problem (1)-(3) can be formulated as $L U=\lambda U$. With this formulation the operator $L$ is independent of $\lambda$.

Now, for an arbitrary $F \in \delta$ we consider $L U-\lambda U=F$, for which the solution $U \in \mathscr{D}$ is given by

$$
U(x)=\int_{0}^{\infty} G(x, \xi, \lambda) F(\xi) d \xi,
$$

where $G(x, \xi, \lambda)$ is the Green's matrix for the problem, and $G(x, \xi, \lambda)$ is the solution of $L G-\lambda G=I$, where

$$
I=\left(\begin{array}{cc}
\delta(x-\xi) & 0 \\
0 & e^{-\xi}
\end{array}\right) .
$$


Here $\delta(x-\xi)$ is the Dirac delta function. That $G$ satisfies this problem follows immediately from the fact that if $(5)$ is to represent the solution of (1)-(3), then it suffices to have

$$
(L-\lambda) U=\int_{0}^{\infty}(L-\lambda) G(x, \xi, \lambda) F(\xi) d \xi=F(x) .
$$

Thus, $(L-\lambda) G$ must be a matrix, say $I$, such that $\int_{0}^{\infty} I F d \xi=F(x)$. A simple calculation shows that $I$ as given by (6) is such a matrix.

Now, our problem is to derive the conditions imposed on $G$ by the requirement that $(L-\lambda) G=I$. Since the differential operator acts on the elements of the subspace $D, G$ must be a $2 \times 2$ matrix, each column of which is an element of $\mathfrak{D}$. Thus, $G$ is given by

$$
G(x, \xi, \lambda)=\left(\begin{array}{cc}
g_{1}(x, \xi, \lambda) & g_{3}(x, \xi, \lambda) \\
g_{2}(\xi, \lambda) & g_{4}(\xi, \lambda)
\end{array}\right)
$$

where $g_{1}$ and $g_{3}$ are bounded as $x \rightarrow \infty$, and

$$
\begin{aligned}
& g_{1}(0, \xi, \lambda)=g_{2}(\xi, \lambda), \\
& g_{3}(0, \xi, \lambda)=g_{4}(\xi, \lambda) .
\end{aligned}
$$

The unique $g_{i}, i=1,2,3,4$, having these properties can be computed by standard techniques [5] and are given by

$$
\begin{aligned}
g_{1}(x, \xi, \lambda) & =\frac{\left(\cos \sqrt{\lambda} x_{<}+\sqrt{\lambda} \sin \sqrt{\lambda} x_{<}\right) e^{i \sqrt{\lambda} x_{>}}}{\lambda-i \sqrt{\lambda}}, \\
g_{2}(\xi, \lambda) & =\frac{e^{i \sqrt{\lambda} \xi}}{\lambda-i \sqrt{\lambda}}, \\
g_{3}(x, \xi, \lambda) & =\frac{-e^{-\xi} e^{i \sqrt{\lambda} x}}{\lambda-i \sqrt{\lambda}}, \\
g_{4}(\xi, \lambda) & =\frac{-e^{-\xi}}{\lambda-i \sqrt{\lambda}} .
\end{aligned}
$$

Here $x_{<}$and $x_{>}$are, respectively, the lesser and greater of $x$ and $\xi$, and the square root is such that $0<\arg \lambda<2 \pi$.

The spectral representation results from showing that

$$
\lim _{R \rightarrow \infty} \frac{1}{2 \pi i} \int_{C_{R}} G(x, \xi, \lambda) d \lambda
$$

converges to $I$. The contour $C_{R}$ is a circle of radius $R$ about the origin in the complex $\lambda$-plane cut along the positive real axis including the origin. It will be convenient to let $\sqrt{\lambda}=l, 0<\arg \lambda<2 \pi$. Then, (11) transforms 
to

$$
\lim _{R \rightarrow \infty} \frac{1}{\pi i} \int_{\Gamma_{R}} G\left(x, \xi, l^{2}\right) l d l
$$

where $\Gamma_{R}$ is a closed contour in the complex $l$-plane consisting of a semicircle of radius $R$ in the upper half-plane together with the real axis from $-R$ to $R$.

It is clear that all four functions $g_{i}(x, \xi, \lambda), i=1, \cdots, 4$, have simple poles at $\lambda=-1$ which transform to simple poles at $l=i$. Now, we assume that the integral along the circular part of the contour $\Gamma_{R}$ tends to $I$ as $R \rightarrow \infty$. Then, by a simple residue calculation we obtain

$$
I \equiv\left(\begin{array}{cc}
\delta(x-\xi) & 0 \\
0 & e^{-\xi}
\end{array}\right)=\left(\begin{array}{ll}
A_{1} & A_{3} \\
A_{2} & A_{4}
\end{array}\right),
$$

where

$$
\begin{aligned}
& A_{1}=\delta(x-\xi)=-2 e^{-\xi} e^{-x} \\
&+\frac{2}{\pi} \int_{0}^{\infty} \frac{(\cos l x+l \sin l x)(\cos l \xi+l \sin l \xi)}{l^{2}+1} d \xi \\
& A_{2}=0=2 e^{-\xi}-\frac{2}{\pi} \int_{0}^{\infty} \frac{(\cos l \xi+l \sin l \xi)}{l^{2}+1} d l, \\
& A_{3}=0=-2 e^{-x} e^{-\xi}+\frac{2}{\pi} e^{-\xi} \int_{0}^{\infty} \frac{(\cos l \xi+l \sin l \xi)}{l^{2}+1} d l \\
& A_{4}=e^{-\xi}=\frac{-2}{\pi} e^{-\xi} \int_{0}^{\infty} \frac{d l}{l^{2}+1}+2 e^{-\xi}
\end{aligned}
$$

Now, let $F$ be an arbitrary vector in $S$; that is,

$$
F(x)=\left(\begin{array}{c}
f(x) \\
f_{0}
\end{array}\right) \text {. }
$$

The spectral representation for $F$ results from the following:

$$
F(x)=\int_{0}^{\infty}\left(\begin{array}{cc}
\delta(x-\xi) & 0 \\
0 & e^{-\xi}
\end{array}\right) F(\xi) d \xi=\int_{0}^{\infty}\left(\begin{array}{ll}
A_{1} & A_{3} \\
A_{2} & A_{4}
\end{array}\right)\left(\begin{array}{c}
f(\xi) \\
f_{0}
\end{array}\right) d \xi .
$$

We substitute (12)-(14) into (16), carry out the matrix multiplication, and obtain, after some straight-forward algebraic manipulation, that our spectral representation is

$$
\begin{aligned}
f(x)=-2 e^{-x}\left[f_{0}+\int_{0}^{\infty} e^{-\xi} f(\xi) d \xi\right] \\
+\frac{2}{\pi} \int_{0}^{\infty} \frac{(\cos l x+l \sin l x)}{l^{2}+1} \\
\cdot\left[f_{0}+\int_{0}^{\infty} f(\xi)(\cos l \xi+l \sin l \xi) d \xi\right] d l
\end{aligned}
$$




$$
\begin{aligned}
f_{0}= & 2\left[f_{0}+\int_{0}^{\infty} e^{-\xi} f(\xi) d \xi\right] \\
& -\frac{2}{\pi} \int_{0}^{\infty} \frac{1}{l^{2}+1}\left[f_{0}+\int_{0}^{\infty} f(\xi)(\cos l \xi+l \sin l \xi) d \xi\right] d l .
\end{aligned}
$$

Thus, we have established (formally) that the right-hand side of (17) is to represent $f(x)$ everywhere in the interval $x>0$, but at $x=0$ it is to become discontinuous in such a way that it represents the arbitrarily prescribed scalar $-f_{0}$. More precisely, we shall now abandon our formalism and rigorously prove the theorem.

TheOREM. Let $f_{0}$ be an arbitrary scalar, and let $f(x)$ be a function which is continuous and which has continuous first and second derivatives in $x>0$. Suppose, further, that

$$
\begin{aligned}
& f^{\prime \prime}(x)=O\left(x^{-2}\right) \text { as } x \rightarrow \infty, \\
& f(x)=O\left(x^{-1}\right) \text { as } x \rightarrow \infty .
\end{aligned}
$$

Then, the representation on the right of (17) converges to $f(x)$ everywhere in $x>0$, and for $x=0$ it converges to $-f_{0}$ as given by (18).

The conditions on $f(x)$ are taken for convenience in simplifying the proof. The theorem can undoubtedly be proved under much weaker restrictions.

Proof. Consider the contour integral $J$ given by

$$
J \equiv\left(\begin{array}{l}
J_{0} \\
J_{1}
\end{array}\right)=\lim _{R \rightarrow \infty} \frac{1}{\pi i} \int_{\Gamma_{R}}\left(\int_{0}^{\infty} G\left(x, \xi, k^{2}\right) F(\xi) d \xi\right) k d k .
$$

Here $\Gamma_{R}$ is a closed contour in the complex $k$-plane consisting of a semicircle $C_{R}$ of radius $R$ in the upper half-plane together with the real axis from $-R$ to $R$, and

$$
G\left(x, \xi, k^{2}\right)=\left(\begin{array}{cc}
g_{1}\left(x, \xi, k^{2}\right) & g_{3}\left(x, \xi, k^{2}\right) \\
g_{2}\left(\xi, k^{2}\right) & g_{4}\left(\xi, k^{2}\right)
\end{array}\right), \quad F(x)=\left(\begin{array}{c}
f(x) \\
f_{0}
\end{array}\right)
$$

where

$$
\begin{aligned}
g_{1}\left(x, \xi, k^{2}\right) & =\frac{\left(\cos k x_{<}+k \sin k x_{<}\right) e^{i k x}>}{k^{2}-i k}, \\
g_{2}\left(\xi, k^{2}\right) & =\frac{e^{i k \xi}}{k^{2}-i k} \\
g_{3}\left(x, \xi, k^{2}\right) & =\frac{-e^{-\xi} e^{i k x}}{k^{2}-i k} \\
g_{4}\left(\xi, k^{2}\right) & =\frac{-e^{-\xi}}{k^{2}-i k}
\end{aligned}
$$


Using (20), we can write the integrals in (19) as

(21) $J_{0}=\lim _{R \rightarrow \infty} \frac{1}{\pi i} \int_{\Gamma_{R}} \int_{0}^{\infty}\left[g_{1}\left(x, \xi, k^{2}\right) f(\xi)+g_{3}\left(x, \xi, k^{2}\right) f_{0}\right] d \xi k d k$

(22) $\quad J_{1}=\lim _{R \rightarrow \infty} \frac{1}{\pi i} \int_{\Gamma_{R}} \int_{0}^{\infty}\left[g_{2}\left(\xi, k^{2}\right) f(\xi)+g_{4}\left(\xi, k^{2}\right) f_{0}\right] d \xi k d k$.

First, we consider (21) for which we can write the integral as

$$
\begin{aligned}
& \frac{1}{\pi i} \int_{\Gamma_{R}} \int_{0}^{\infty}\left[g_{1}\left(x, \xi, k^{2}\right) f(\xi)\right.\left.+g_{3}\left(x, \xi, k^{2}\right) f_{0}\right] d \xi k d k \\
&(23)=\frac{1}{\pi i} \int_{-\infty}^{\infty} \Phi_{1}(x, k) d k+\frac{1}{\pi i} \int_{C_{R}} \Phi_{1}(x, k) d k+\frac{1}{\pi i} \int_{-\infty}^{\infty} \Phi_{2}(x, k) d k \\
& \quad+\frac{1}{\pi i} \int_{C_{R}} \Phi_{2}(x, k) d k-\frac{f_{0}}{\pi i} \int_{\Gamma_{R}} \frac{e^{i k x}}{k-i} d k,
\end{aligned}
$$

where

$$
\begin{aligned}
& \Phi_{1}(x, k)=\frac{k e^{i k x}}{k^{2}-i k} \int_{0}^{x}(\cos k \xi+k \sin k \xi) f(\xi) d \xi \\
& \Phi_{2}(x, k)=\frac{k(\cos k x+k \sin k x)}{k^{2}-i k} \int_{x}^{\infty} e^{i k \xi} f(\xi) d \xi .
\end{aligned}
$$

We now estimate the second and fourth integrals in (23). Integrating twice by parts in (24), we find that on $C_{R}$

$$
\begin{aligned}
& \Phi_{1}(x, k)=\frac{f(x) e^{i k x} \sin k x}{k(k-i)}-\frac{f(x) e^{i k x} \cos k x}{k-i} \\
&+\frac{f(0) e^{i k x}}{k-i}+O\left(\frac{1}{k^{2}}\right), \quad \operatorname{Im}\{k\}>0, \quad x>0,
\end{aligned}
$$

and

$$
\begin{aligned}
\Phi_{2}(x, k)= & \frac{i f(x) e^{i k x} \cos k x}{k(k-i)} \\
& +\frac{i f(x) \sin k x e^{i k x}}{k-i}+O\left(\frac{1}{k^{2}}\right), \quad \operatorname{Im}\{k\}>0, \quad x>0 .
\end{aligned}
$$

Thus, for the second and fourth integrals in (23) we can write

$$
\begin{aligned}
& \frac{1}{\pi i} \int_{C_{R}}\left(\Phi_{1}+\Phi_{2}\right) d k \\
& =\frac{-f(x)}{\pi i} \int_{C_{R}} \frac{d k}{k-i}+\frac{f(x)}{\pi} \int_{C_{R}} \frac{d k}{k(k-i)} \\
& \quad+\frac{f(0)}{\pi i} \int_{C_{R}} \frac{e^{i k x}}{k-i} d k+\frac{1}{\pi i} \int_{C_{R}} O\left(\frac{1}{k^{2}}\right) d k
\end{aligned}
$$


The last three integrals in (25) approach zero as $R \rightarrow \infty$, and the first integral on the right-hand side of (25) approaches $i \pi$ as $R \rightarrow \infty$. Hence,

$$
\lim _{R \rightarrow \infty} \frac{1}{\pi i} \int_{C_{R}}\left(\Phi_{1}+\Phi_{2}\right) d k=-f(x) .
$$

Therefore, from (23) and (26) we have

$$
J_{0}=-f(x)-2 f_{0} e^{-x}+\frac{1}{\pi i} \int_{-\infty}^{\infty}\left(\Phi_{1}+\Phi_{2}\right) d k .
$$

Now,

$$
\begin{aligned}
\frac{1}{\pi i} \int_{-\infty}^{\infty}\left(\Phi_{1}+\Phi_{2}\right) d k=\frac{1}{\pi i} \int_{-\infty}^{0} \Phi_{1} d k+ & \frac{1}{\pi i} \int_{0}^{\infty} \Phi_{1} d k \\
& +\frac{1}{\pi i} \int_{-\infty}^{0} \Phi_{2} d k+\frac{1}{\pi i} \int_{0}^{\infty} \Phi_{2} d k .
\end{aligned}
$$

Let $k=-l$ in the first and third integrals on the right-hand side of (28), and let $k=+l$ in the second and fourth integrals. Then, after some algebraic manipulation, we obtain

$$
\begin{aligned}
\frac{1}{\pi i} \int_{-\infty}^{\infty}\left(\Phi_{1}+\Phi_{2}\right) & d k \\
= & \frac{2}{\pi} \int_{0}^{\infty} \frac{(\cos l x+l \sin l x)}{l^{2}+1} \int_{0}^{\infty} f(\xi)(\cos l \xi+l \sin l \xi) d \xi d l .
\end{aligned}
$$

Thus, (27) can be written as

$$
\begin{aligned}
J_{0}=- & f(x)-2 f_{0} e^{-x} \\
& +\frac{2}{\pi} \int_{0}^{\infty} \frac{(\cos l x+l \sin l x)}{l^{2}+1} \int_{0}^{\infty} f(\xi)(\cos l \xi+l \sin l \xi) d \xi d l .
\end{aligned}
$$

We now evaluate $J_{0}$, defined by (21), by the theory of residues. Clearly, the only singularity of the integrand (in the integral with respect to $k$ ) occurs as a simple pole at $k=i$. After some straightforward algebraic manipulation we find that

$$
J_{0}=2 e^{-x} \int_{0}^{\infty} f(\xi) e^{-\xi} d \xi-2 f_{0} e^{-x} .
$$

Upon combining (29) and (30) and using the fact that

$$
2 e^{-x}=\frac{2}{\pi} \int_{0}^{\infty} \frac{(\cos l x+l \sin l x)}{l^{2}+1} d l,
$$

the representation (17) immediately follows. We proved the validity of (17) by starting with (21); similarly, the representation (18) follows by a completely analogous proof starting with a consideration of (22). 
We shall find it convenient to write the representations (17), (18) as a "transform pair" in the following way:

$$
\begin{gathered}
f(x)=-2 \beta e^{-x}+\frac{2}{\pi} \int_{0}^{\infty} B(k) \frac{(\cos k x+k \sin k x)}{k^{2}+1} d k, \\
-f_{0}=-2 \beta+\frac{2}{\pi} \int_{0}^{\infty} B(k) \frac{1}{k^{2}+1} d k,
\end{gathered}
$$

where

$$
\begin{gathered}
\beta=f_{0}+\int_{0}^{\infty} e^{-\xi} f(\xi) d \xi, \\
B(k)=f_{0}+\int_{0}^{\infty} f(\xi)(\cos k \xi+k \sin k \xi) d \xi .
\end{gathered}
$$

Before applying (31), (32) to a boundary value problem, we wish to make several observations and comments on the representation and the above theorem. Notice that the spectral representation consists of the simultaneous integral representation of both an arbitrary function $f(x)$ and an arbitrary scalar $f_{0}$. Moreover, the representation (i.e., the righthand side of (17)) represents $f(x)$ on the interval $x>0$, and in addition it becomes discontinuous in a prescribed manner in order to converge to $-f_{0}$ at $x=0$. Although this is unusual, such behavior is not new. Indeed, Langer [6] found the same phenomenon for certain series of eigenfunctions, and he references even earlier works where similar things occur. Nevertheless, it is interesting to observe that we do not obtain the usual Fouriertype convergence to $\frac{1}{2}[f(x+0)+f(x-0)]$ at points of discontinuity of $f(x)$.

In the matter of the expansion of arbitrary functions in series of eigenfunctions, the theory of linear, first order, ordinary differential systems includes theorems asserting that $n$ such functions may be simultaneously expanded with a single determination of coefficients. In 1929 Langer [7] showed that the same is true for a single $n$th order nonsingular equation; before this the expansion theorems for such problems concerned only the expansion of a single function. Thus, the occurrence of two simultaneous expansions for our second order problem is not surprising. Our problem, however, is singular, and thus, Langer's theory is not applicable. Furthermore, reflecting the fact that (1)-(3) is a singular problem, it is not surprising that we obtain integral transforms rather than eigenfunction expansions.

3. Solution of a typical boundary value problem. The eigenvalue problem (1)-(3) can arise in a variety of physical problems. One of the simplest is the following: A semi-infinite right cylindrical solid with cross section of 
arbitrary shape and size and with plane terminal face at $x=0$ has its lateral surface insulated against the passage of heat and has an initial distribution of temperature depending only on the longitudinal coordinate $x$. A liquid of arbitrary initial temperature is kept well stirred to insure at each instant a uniform temperature throughout it, and at time $t=0$ the plane terminal face of the solid is placed in contact with this liquid. For simplicity we assume that the liquid is not accessible to a transfer of heat to or from its surrounding medium. Our problem is to determine the temperature of the liquid and the distribution of temperature in the solid at any time $t>0$.

The temperature $w(x, t)$ of the solid at position $x$ at time $t$ is governed by the heat equation

$$
\alpha^{2} w_{x x}=w_{t} .
$$

Here $\alpha^{2}=k / \rho c$, where $k$ is the conductivity of the solid, $\rho$ and $c$ are its density and specific heat, respectively. Let $A$ be the cross-sectional area of the solid, and let $x=0$ denote the position of its terminal face, which is in contact with the liquid. Then, if $v(t)$ denotes the temperature of the liquid at time $t$, the thermal contact between the solid and the liquid implies the relation

$$
w(0, t)=v(t), \quad t>0 .
$$

The rate at which heat flows from the solid to the liquid is $-k A u_{x}$ at $x=0$, and the rate of accumulation of heat in the liquid is $q M v^{\prime}(t)$, where $M$ and $q$ are respectively the mass and specific heat of the liquid. Thus, the solution must also satisfy the condition that

$$
q M v^{\prime}(t)=-k A w_{x}(0, t) .
$$

Finally, if $w_{0}(x)$ and $v_{0}$ denote respectively the initial temperatures in the solid and liquid, it is clear that we must have

$$
\begin{aligned}
\lim _{t \rightarrow 0} w(x, t) & =w_{0}(x), \quad x>0, \\
\lim _{t \rightarrow 0} v(t) & =v_{0} .
\end{aligned}
$$

The problem as formulated in (34)-(38) involves both $w(x, t)$ and $v(t)$. We may formulate a problem for $w(x, t)$ alone as follows. First, let us write (36) as

$$
\frac{\sigma}{\alpha^{2}} v^{\prime}(t)+w_{x}(0, t)=0,
$$

where $\sigma=q M \alpha^{2} / k A$. Without loss of generality we can take $\sigma=1$. Then, 
we can use (35) to write (39) as

$$
w_{\iota}(0, \iota)+\alpha^{2} w_{.}(0, t)=0 .
$$

Thus, the problem for $w(x, t)$ is

$$
\begin{aligned}
\alpha^{2} w_{x x} & =w_{t}, \quad x>0, \quad t>0, \\
w_{t}(0, t)+\alpha^{2} w_{x}(0, t) & =0, \quad t>0, \\
w(x, 0) & =w_{0}(x), \quad x>0, \\
w(0,0) & =v_{0} .
\end{aligned}
$$

In keeping with the physics of the problem, we are clearly assuming that the boundary condition at $x=\infty$ is that the solution $w(x, t)$ remain bounded.

The problem (40)-(43) can be solved easily by a straight-forward application of the Laplace transform in time. (We do this briefly later.) Our goal here, however, is to show that the classical separation methods can be extended so that they are also applicable. It is the appearance of the derivative with respect to time in (41) that precludes an immediate application of these methods; for as we shall now see, it is because of this derivative that the eigenvalue parameter appears in the boundary condition associated with the separated ordinary differential equation.

If, as is usual, we assume a product solution of the form $w(x, t)$ $=u(x) g(t)$, then (40) and (41) become

$$
\begin{aligned}
& \frac{u^{\prime \prime}(x)}{u(x)}=\frac{g^{\prime}(t)}{\alpha^{2} g(t)}=-\lambda, \\
& \frac{u^{\prime}(0)}{u(0)}=\frac{-g^{\prime}(t)}{\alpha^{2} g(t)}=\lambda,
\end{aligned}
$$

where we have taken $-\lambda$ as the separation constant. Therefore, we are immediately led to the problem of finding the spectral representation associated with the second order system (1)-(3) which we treated in $\$ 2$.

By using the representation derived in that section we now solve our problem (40)-(43). It is clear that a solution of (40) satisfying (41) is given by

$$
w(x, t)=-2 \beta e^{-x+\alpha^{2} t}+\frac{2}{\pi} \int_{0}^{\infty} B(k)\left[\frac{\cos k x+k \sin k x}{k^{2}+1}\right] e^{-\alpha^{2} k^{2} t} d k .
$$

Now, $\beta$ and $B(k)$ must be determined so that the conditions (42) and (43) are both satisfied. Thus, we must have

$$
w_{0}(x)=-2 \beta e^{-x}+\frac{2}{\pi} \int_{0}^{\infty} B(k)\left[\frac{\cos k x+k \sin k x}{k^{2}+1}\right] d k,
$$


and

$$
v_{0}=-2 \beta+\frac{2}{\pi} \int_{0}^{\infty} B(k) \frac{1}{k^{2}+1} d k .
$$

Hence, the simultaneous relations (45) and (46) require that the righthand side of (45) shall, in the usual way, represent a prescribed function in the interval $x>0$, but in addition it shall become discontinuous in a prescribed manner at the endpoint $x=0$. Therefore, the initial temperature of the liquid influences the solution only in the prescription of the discontinuity, a fact which is clearly obvious on physical grounds.

From (31)-(34) we can immediately determine $\beta$ and $B(k)$ as

$$
\begin{gathered}
\beta=-v_{0}+\int_{0}^{\infty} e^{-\xi} w_{0}(\xi) d \xi \\
B(k)=-v_{0}+\int_{0}^{\infty} w_{0}(\xi)(\cos k \xi+k \sin k \xi) d k .
\end{gathered}
$$

Inserting (47) and (48) into (44), we find that the solution of the boundary value problem $(40)-(43)$ is

$$
\begin{aligned}
& w(x, t)= 2 v_{0} e^{-x+\alpha^{2} t}-2 e^{-x+\alpha^{2} t} \int_{0}^{\infty} e^{-\xi} w_{0}(\xi) d \xi \\
&-\frac{2 v_{0}}{\pi} \int_{0}^{\infty} e^{-\alpha^{2} k^{2} t}\left[\frac{\cos k x+k \sin k x}{k^{2}+1}\right] d k \\
&+\frac{2}{\pi} \int_{0}^{\infty} e^{-\alpha^{2} k^{2} t}\left[\frac{\cos k x+k \sin k x}{k^{2}+1}\right] \\
& \cdot \int_{0}^{\infty} w_{0}(\xi)(\cos k \xi+k \sin k \xi) d \xi d k .
\end{aligned}
$$

It is interesting to briefly consider the standard Laplace transform approach to problems like ours. In the standard manner, we find that the solution of $(40)-(43)$ is

$$
\begin{aligned}
& w(x, t)=\frac{1}{2 \pi i} \int_{\gamma-i \infty}^{\gamma+i \infty} \frac{v_{0}}{s-\alpha \sqrt{s}} e^{(-\sqrt{s} / \alpha) x+s t} d s \\
& \quad-\frac{1}{2 \pi i \alpha^{2}} \int_{\gamma-i \infty}^{\gamma+i \infty} e^{s t} \int_{0}^{\infty} \Gamma(x, \xi, s) w_{0}(\xi) d \xi d s,
\end{aligned}
$$

where

(51) $\Gamma(x, \xi, s)=\frac{-\alpha}{2 \sqrt{s}} e^{(-\sqrt{s} / \alpha) x_{>}}\left[e^{(\sqrt{s} / \alpha) x_{1}}-\left(\frac{s+\alpha \sqrt{s}}{s-\alpha \sqrt{s}}\right) e^{(-\sqrt{s} / \alpha) x_{<}}\right]$, 
and, as usual, the contour of integration in the $s$-plane is a line parallel to the imaginary axis and to the right of all singularities of the integrand. In (50) and (51) the square root is such that $-\pi<\arg s<\pi$, a fact which easily follows from the requirement that the solution is bounded at infinity. When left in the form (50), the solution is not particularly useful either for its physical interpretation or for numerical purposes. However, other forms can be obtained from (50) by closing the contour in the lefthalf of the s-plane (and taking account of the branch cut along the negative real axis). After a considerable amount of straightforward analysis we can reduce $(50)$ to $(49)$, which is certainly a more desirable representation of the solution than (50).

\section{REFERENCES}

[1] D. S. Cohen, Eigenfunction expansions and non-selfadjoint boundary value problems, Comm. Pure Appl. Math., 17 (1964), pp. 1-22.

[2] - - Separation of variables and alternative representations for non-selfadjoint boundary value problems, Ibid., 17 (1964), pp. 23-24.

[3] - New eigenfunction expansions and alternative representations for the reduced wave equation, J. Math. Mech., 14 (1965), pp. 403-411.

[4] R. V. Churchild, Extensions of operational mathematics, Proceedings of the Conference on Differential Equations, University of Maryland, College Park, 1956, pp. 235-250.

[5] B. Friedman, Principle and Techniques of Applied Mathematics, John Wiley, New York, 1956.

[6] R. E. Langer, A problem in diffusion or in the flow of heat for a solid in contact with a fluid, Tôhoku Math. J., 35 (1935), pp. 260-275.

[7] - The expansion problem in theory of ordinary linear differential systems of the second order, Trans. Amer. Math. Soc., 31 (1929), pp. 868-906. 